\title{
openheart Four pillars of heart failure: contemporary pharmacological therapy for heart failure with reduced ejection fraction
}

\author{
Sam Straw (D) , ${ }^{1}$ Melanie McGinlay, ${ }^{2}$ Klaus K Witte ${ }^{1}$
}

To cite: Straw S, McGinlay M, Witte KK. Four pillars of heart failure: contemporary pharmacological therapy for heart failure with reduced ejection fraction. Open Heart 2021;8:e001585. doi:10.1136/ openhrt-2021-001585

Accepted 11 February 2021

Check for updates

(c) Author(s) (or their employer(s)) 2021. Re-use permitted under CC BY. Published by BMJ.

${ }^{1}$ Leeds Institute of Cardiovascular and Metabolic Medicine, University of Leeds, Leeds, UK

${ }^{2}$ Cardiorespiratory Clinical Services Unit, Leeds Teaching Hospitals NHS Trust, Leeds, UK

Correspondence to Dr Klaus K Witte; k.k.witte@ leeds.ac.uk

\section{INTRODUCTION}

The past two decades have heralded dramatic improvements in outcomes for people living with heart failure with reduced ejection fraction (HFrEF). ${ }^{1}$ The more widespread implementation of disease modifying pharmacological therapies, ${ }^{2}$ supported by landmark trials of renin-angiotensin system inhibitors ${ }^{3}$ and beta-blockers ${ }^{4}$ have improved longevity despite a background of an ageing and increasingly multimorbid population. Although the benefits of comprehensive pharmacological therapies are clear, the real-world attainment of target doses ${ }^{56}$ and utilisation of novel agents such as angiotensin receptor-neprilysin inhibitors (ARNI) ${ }^{7}$ remain low. Furthermore, HFrEF remains a disease associated with significant morbidity and reduced survival relative to those without HFrEF, even after taking into account comorbidities. ${ }^{8}$ Recently, trials have demonstrated improved outcomes in people with HFrEF receiving sodium-glucose co-transporter 2 inhibitors (SGLT2i). ${ }^{9}{ }^{10}$ However, it is currently unclear how these agents will be used alongside established therapies. Now is therefore an opportune moment to pause and reflect on our current practice, barriers to further progress and how future guidelines might work better for our patients. In this viewpoint we summarise how our current linear approach, on a background of increasingly complex pharmacotherapy has the potential to cause confusion and consequent delays which could lead to even worse attainment of optimal therapies. On the other hand, a more parallel approach to the initiation and optimisation of the Four Pillars of Heart Failure would simplify our approach, yielding benefits for our patients and healthcare systems.

\section{HOW DID WE GET HERE?}

Heart failure guidelines are based around inhibition of the renin-angiotensin and sympathetic nervous systems, two fundamental pathways which drive the pathophysiology of HFrEF using ACE inhibitors (ACEi) and beta-blockers. In both European ${ }^{2}$ and American guidelines ${ }^{11}$ additional therapies are recommended for patients who 'remain symptomatic' with persistently impaired left ventricular (LV) function despite maximally tolerated doses of ACEi and beta-blockers. These guidelines differ subtly regarding the timing of mineralocorticoid receptor antagonists (MRA) relative to other therapies but are otherwise broadly similar, advocating a linear approach. This attempts to avoid 'unnecessary' treatments in patients who 'respond' but has several important limitations. First, while guidelines do not stipulate a time interval between alterations to therapy, the need for further assessment and re-evaluation of LV function inevitably results in delays initiating additional agents as well as contributing further follow-up and imaging costs. In clinical practice it typically takes many months before patients receive optimised doses of these medications, and many never do, even where integrated hospital and community care is available. ${ }^{5}$ Second, the barrier of 'response' is confusing and misplaced: does 'response' mean asymptomatic or merely improved? In our experience, while patients often feel better, they rarely become asymptomatic (NYHA (New York Heart Association) class 1$),{ }^{12}$ an observation supported by real-world data even in those receiving ARNI. ${ }^{13} 14$ Moreover, we should consider whether a highly subjective and poorly reproducible assessment is appropriate to determine our allocation of life-saving treatments. ${ }^{15}$ Hence, criteria 
requiring repeat assessment act as a barrier to initiating additional therapies such as MRA or ARNI, ${ }^{7}$ which are regarded as 'second-line' due to the hierarchical framework which places greater emphasis on therapies based on the chronological sequence in which the trials were performed. There is no logical basis to assume that drug classes trialled earliest would be the most beneficial, yet this is what guidelines imply. Therefore, if we are to make progress, future guidelines must address these limitations and incorporate the Four Pillars of Heart Failure into a comprehensive disease modifying programme for all people living with HFrEF.

\section{LEARNING LESSONS FROM ARNI}

The PARADIGM-HF trial showed that a combination of angiotensin receptor blocker and a neprilysin inhibitor (sacubitril-valsartan) was superior to an ACEi in preventing cardiovascular deaths or hospitalisation for heart failure (HR 080, 95\% CI 0.73 to 0.87 ) and reducing all-cause mortality ( $\mathrm{HR} 0.84$, 95\% CI 0.76 to 0.93). ${ }^{16}$ Despite overwhelming efficacy which led to the trial being stopped early, the utilisation of ARNI in the real-world has been suboptimal, with less than $1 \%$ penetration in eligible patients. The reluctance of physicians to prescribe ARNI may in part be that, unusually, the recommendations are based on a single trial, studied in an 'A+Bvs C' fashion. Furthermore, the comparator was a submaximal dose of enalapril compounded by lower blood pressure in those allocated ARNI suggesting undertreatment in the control arm. ${ }^{16}$ It has also been suggested that the trial was additionally biased in favour of the novel agent due to a double drug run-in period of unequal times, in which those randomised to ARNI had already received an ACEi and were therefore pre-selected (20\% of patients were lost during the run-in period). ${ }^{17}$ Drug doses are related to outcomes in HFrEF ${ }^{18-20}$ and the HR for the composite outcome in PARADIGM-HF between sacubitril-valsartan and enalapril was similar to the comparison of high and low dosing of lisinopril in the ATLAS trial. ${ }^{21}$ However, post-hoc analysis has shown that the point estimates for the benefit of low dose ARNI compared with low dose ACEi were identical to the point estimate of the overall trial, ${ }^{22}$ and real-world data have shown clear improvements in outcomes, symptoms and quality of life compared with standard of care ACEi. ${ }^{23}$

Another difficulty employing ARNI across the board includes the wash-out period required following cessation of ACEi due to risks of angioedema. Although not insurmountable, such requirements challenge heart failure programmes facing reduced face-to-face appointments due to service redesign and the current pandemic. Hence, if the benefits of the activity are perceived (whether correctly or incorrectly) to be minimal, physician inertia may prevail. To counter this, initiating ARNI at the point of diagnosis would mitigate the risk of inertia while also providing a more effective treatment to patients during the period of highest risk.

\section{IMPLEMENTING NOVEL AGENTS INTO HEART FAILURE}

\section{PATHWAYS}

The efficacy of SGLT2i in addition to standard therapies for people with HFrEF has been confirmed with consistent and near identical $25 \%$ risk reduction of the primary end point of cardiovascular death or hospitalisation for heart failure from both dapagliflozin and empagliflozin. ${ }^{10} 24$ Both trials also demonstrated a slow of decline in renal function; EMPEROR-Reduced showed a $50 \%$ relative risk reduction for the composite renal endpoint ${ }^{10}$ (although this was non-significant in DAPA-HF). ${ }^{24}$

It is anticipated that more than four out of five people with HFrEF in contemporary registries ${ }^{25}$ will be eligible based on the inclusion criteria of these trials. The beneficial effects on renal outcomes are particularly attractive in a disease process typically associated with progressive decline of kidney function which often prevents the initiation or intensification of renin-angiotensin system inhibitors. Furthermore, SGLT2i are safe, with a low incidence of serious side effects (no patients without diabetes developed ketoacidosis in DAPA-HF or EMPEROR-Reduced), a lack of dosing considerations and minimal effects on blood pressure.

Given the somewhat simpler approach taken in trials of SGLT2i, the ease of use and clear benefits, it is likely that uptake among physicians will be enthusiastic, although it is yet unclear how these agents might fit into our current practice. There is a risk that SGLT2i become an additional agent for patients who do not 'respond' with conventional pharmacological therapy, rather than a fundamental Pillar of Heart Failure. The totality of the available evidence suggests the benefits of SGLT2i are consistent across subgroups, including diabetes status, baseline ARNI and symptoms. SGLT2i must therefore be regarded as a unique class of medication with a novel mechanism of action to be used in all eligible patients.

\section{THE POSSIBILITIES OF A COMPREHENSIVE APPROACH}

The four drug classes are complementary to each other and cross-trial comparisons have shown that a comprehensive disease modification strategy beyond the treatments which most patients receive (ACEi and betablocker) with ARNI, MRA and SGLT2i are associated with improved outcomes. A typical patient aged 65 years can expect to live an additional 5 years if receiving a comprehensive strategy with the Four Pillars, compared with conventional therapy. ${ }^{26}$

\section{INERTIA: KNOW YOUR ENEMY}

Drugs, trial design and side effects aside, the key obstacle to therapy intensification is physician inertia in patients who are deemed to have stabilised or 'responded' to treatment. For some with a recent decompensation this might be appropriate, ${ }^{27} 28$ but the relevant clinical trials were carried out in ambulatory patients receiving stable doses of previous generations of medical therapy, most 


\section{The Four Pillars of Heart Failure}

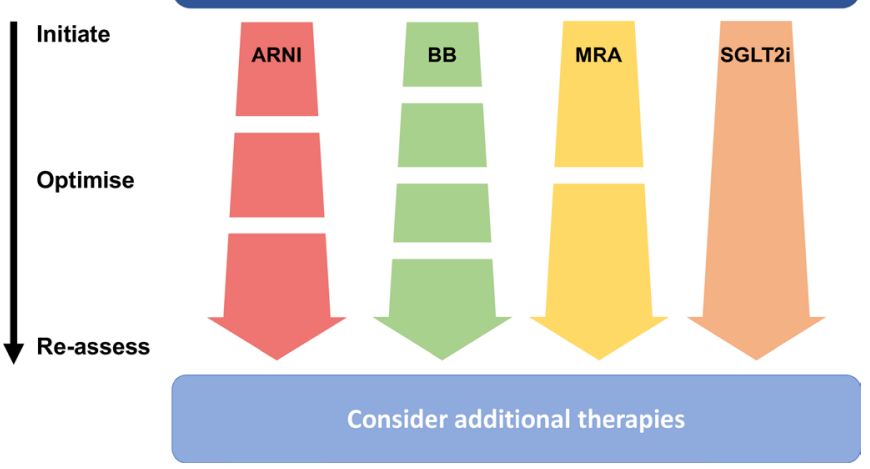

Figure 1 Initiation and optimisation of the Four Pillars of Heart Failure. All agents are initiated in parallel. This is followed by up-titration in one, two or three steps, as required. Additional therapies are then considered as a final step. ARNI, angiotensin receptor-neprilysin inhibitors; $\mathrm{BB}$, beta-blocker; MRA, mineralocorticoid receptor antagonists;SGLT2i, sodium-glucose co-transporter 2inhibitors.

of which had class II symptoms. ${ }^{101624}$ Of particular relevance to SGLT2i, a striking result from DAPA-HF was the early benefit from dapagliflozin, with a reduction in worsening heart failure events observed within 28 days. $^{24}$

\section{SIMPLIFY TO PROGRESS}

We propose a novel conceptual framework for the implementation of pharmacological therapies in HFrEF, in which the Four Pillars of Heart Failure are introduced in parallel, very early in the patient pathway with subsequent optimisation of dosing where required (figure 1). ${ }^{24}$ The rate of dose increments can be tailored to the patient and the service. For most patients, low dose ARNI and SGLT2i could be started simultaneously, followed within a few days by low dose beta-blocker and MRA, followed by up-titration. While others have suggested that initiation with beta-blocker alongside SGLT2i might be more optimal, ${ }^{29}$ we believe that the exact sequent of initiation is unimportant, as long as all Four Pillars are introduced within the first few weeks of diagnosis-although with the lack of dosing considerations and clear evidence from randomised trials of an early beneficial effect, ${ }^{27}$ SGLT2i are an obvious first choice.

This approach is at odds with current practice and a fundamental shift away from the linear approach advocated by guidelines, including the recent technology appraisal of dapagliflozin from the UK. ${ }^{30}$ Nevertheless, it remains the case, that very few patients become asymptomatic with even optimal doses of disease-modifying therapy suggesting that slow up-titration, followed by a decision to embark on the next step based on symptoms is folly.

It seems likely that impairment of renal function following up-titration of ARNI and MRA will become lesser concerns following the introduction of SGLT2i into care pathways, however, real-world data are vital to confirm the safety and feasibility of this approach. Those caring for people with heart failure must be cognisant that early increases in creatinine with SGLT2i are transient and be reassured that in the long-term the less rapid decline in renal function in patients receiving SGLT2i will allow more complete renin-angiotensin system blockade.

\section{CONCLUSION}

The introduction of SGLT2i to the treatment of HFrEF is a chance for us to revisit whether current guidelines for the treatment of HFrEF are fit for purpose. Many patients have waited for weeks or months before ever seeing a cardiologist and receiving a diagnosis, and further delays to treatments have the potential to cause great harm. Moving forwards, we must recognise heart failure for what it is, an incurable disease with a mortality rate similar to many forms of cancer, where any delays cost lives. Once we have done so we need to implement pathways that offer rapid initiation and, where required, up-titration of life-extending therapies.

\section{Twitter Sam Straw @DrSamStraw}

Contributors All authors contributed equally.

Funding This work was supported by the British Heart Foundation: $\mathrm{CH} / 13 / 1 / 30086$.

Competing interests KKW has received speakers' fees and honoraria from Medtronic, Cardiac Dimensions, Novartis, Abbott, BMS, Pfizer, Bayer and has received an unconditional research grant from Medtronic.

Patient consent for publication Not required.

Provenance and peer review Not commissioned; externally peer reviewed.

Data availability statement No data are available.

Open access This is an open access article distributed in accordance with the Creative Commons Attribution 4.0 Unported (CC BY 4.0) license, which permits others to copy, redistribute, remix, transform and build upon this work for any purpose, provided the original work is properly cited, a link to the licence is given, and indication of whether changes were made. See: https://creativecommons.org/ licenses/by/4.0/.

ORCID iD

Sam Straw http://orcid.org/0000-0002-2942-4574

\section{REFERENCES}

1 Cubbon RM, Gale CP, Kearney LC, et al. Changing characteristics and mode of death associated with chronic heart failure caused by left ventricular systolic dysfunction: a study across therapeutic eras. Circ Heart Fail 2011;4:396-403.

2 Ponikowski P, Voors AA, Anker SD, et al. 2016 ESC Guidelines for the diagnosis and treatment of acute and chronic heart failure: The Task Force for the diagnosis and treatment of acute and chronic heart failure of the European Society of Cardiology (ESC)Developed with the special contribution of the Heart Failure Association (HFA) of the ESC. Eur Heart J 2016;37:2129-200.

3 SOLVD Investigators, Yusuf S, Pitt B, et al. Effect of enalapril on survival in patients with reduced left ventricular ejection fractions and congestive heart failure. N Engl J Med 1991;325:293-302.

4 The cardiac insufficiency bisoprolol study II (CIBIS-II): a randomised trial. Lancet 1999;353:9-13.

5 Greene SJ, Fonarow GC, DeVore AD, et al. Titration of medical therapy for heart failure with reduced ejection fraction. J Am Coll Cardiol 2019;73:2365-83.

6 Brunner-La Rocca H-P, Linssen GC, Smeele FJ, et al. Contemporary Drug Treatment of Chronic Heart Failure With Reduced Ejection Fraction: The CHECK-HF Registry. JACC Heart Fail 2019;7:13-21. 
7 Greene SJ, Butler J, Albert NM, et al. Medical Therapy for Heart Failure With Reduced Ejection Fraction: The CHAMP-HF Registry. $J$ Am Coll Cardiol 2018;72:351-66.

8 Drozd M, Relton SD, Walker AMN, et al. Association of heart failure and its comorbidities with loss of life expectancy. Heart 2020 doi:10.1136/heartjnl-2020-317833. [Epub ahead of print: 05 Nov 2020].

9 Colombo G, Casella R, Cazzaniga A, et al. Dapagliflozin in patients with heart failure and reduced ejection fraction. Intern Emerg Med 2020;15:515-7.

10 Packer M, Anker SD, Butler J, et al. Cardiovascular and renal outcomes with Empagliflozin in heart failure. N Engl J Med 2020;383:1413-24.

11 Yancy CW, Jessup M, Bozkurt B, et al. 2017 ACC/AHA/HFSA focused update of the 2013 ACCF/AHA guideline for the management of heart failure: a report of the American College of Cardiology/American heart association Task force on clinical practice guidelines and the heart failure Society of America. Circulation 2017;136:e137-61.

12 Koshy AO, Gallivan ER, McGinlay M, et al. Prioritizing symptom management in the treatment of chronic heart failure. ESC Heart Fail 2020;7:2193-207.

13 Polito MV, Silverio A, Rispoli A, et al. Clinical and echocardiographic benefit of Sacubitril/Valsartan in a real-world population with HF with reduced ejection fraction. Sci Rep 2020;10:6665.

14 Lau CW, Martens P, Lambeets S, et al. Effects of sacubitril/valsartan on functional status and exercise capacity in real-world patients. Acta Cardiol 2019;74:405-12.

15 Blacher M, Zimerman A, Engster PHB, et al. Revisiting heart failure assessment based on objective measures in NYHA functional classes I and II. Heart 2020. doi:10.1136/heartjnl-2020-317984. [Epub ahead of print: 23 Dec 2020]

16 McMurray JJV, Packer M, Desai AS, et al. Angiotensin-neprilysin inhibition versus enalapril in heart failure. $N$ Engl $J$ Med 2014;371:993-1004.

17 Haslam A, Prasad V. Confirmatory trials for drugs Approved on a single trial. Circ Cardiovasc Qual Outcomes 2019;12:e005494.

18 Witte KK, Drozd M, Walker AMN, et al. Mortality reduction associated with $\beta$-adrenoceptor inhibition in chronic heart failure is greater in patients with diabetes. Diabetes Care 2018;41:136-42.

19 Straw S, McGinlay M, Relton SD, et al. Effect of disease-modifying agents and their association with mortality in multi-morbid patients with heart failure with reduced ejection fraction. ESC Heart Fail 2020;7:3859-70.
20 Bristow MR, Gilbert EM, Abraham WT, et al. Carvedilol produces dose-related improvements in left ventricular function and survival in subjects with chronic heart failure. MOCHA Investigators. Circulation 1996;94:2807-16.

21 Packer M, Poole-Wilson PA, Armstrong PW, ATLAS study group. Comparative effects of low and high doses of the angiotensinconverting enzyme inhibitor, lisinopril, on morbidity and mortality in chronic heart failure. Circulation 1999;100:2312-8.

22 Vardeny O, Claggett B, Packer M, et al. Efficacy of sacubitril/ valsartan vs. enalapril at lower than target doses in heart failure with reduced ejection fraction: the PARADIGM-HF trial. Eur $\mathrm{J}$ Heart Fail 2016;18:1228-34.

23 Khariton Y, Fonarow GC, Arnold SV, et al. Association Between Sacubitril/Valsartan Initiation and Health Status Outcomes in Heart Failure With Reduced Ejection Fraction. JACC Heart Fail 2019;7:933-41.

24 McMurray JJV, Solomon SD, Inzucchi SE, et al. Dapagliflozin in patients with heart failure and reduced ejection fraction. $N$ Engl $J$ Med 2019;381:1995-2008.

25 Sharma A, Zhao X, Hammill BG, et al. Trends in Noncardiovascular comorbidities among patients hospitalized for heart failure: insights from the get with the Guidelines-Heart failure registry. Circ Heart Fail 2018:11:e004646.

26 Vaduganathan M, Claggett BL, Jhund PS, et al. Estimating lifetime benefits of comprehensive disease-modifying pharmacological therapies in patients with heart failure with reduced ejection fraction: a comparative analysis of three randomised controlled trials. Lancet 2020;396:121-8.

27 Bhatt DL, Szarek M, Steg PG, et al. Sotagliflozin in patients with diabetes and recent worsening heart failure. $N$ Engl J Med 2021:384:117-128:117-28.

28 Wachter R, Senni M, Belohlavek J, et al. Initiation of sacubitril/ valsartan in haemodynamically stabilised heart failure patients in hospital or early after discharge: primary results of the randomised TRANSITION study. Eur J Heart Fail 2019;21:998-1007.

29 McMurray JJV, Packer M. How should we sequence the treatments for heart failure and a reduced ejection fraction? A redefinition of evidence-based medicine. Circulation 2020;100.

30 National Institute of Health and Care Excelence. Dapagliflozin for treating chronic heart failure with reduced ejection fraction, 2020. Available: https://www.nice.org.uk/guidance/gid-ta10560/documents/ final-appraisal-determination-document [Accessed 08 Feb 2021]. 